Garz, Jona

\title{
"Schriftproben von schwachsinnigen resp. idiotischen Kindern". Testwissen zwischen Psychiatrie und Pädagogik um 1900
}

Reh, Sabine [Hrsg.]; Bühler, Patrick [Hrsg.]; Hofmann, Michèle [Hrsg.]; Moser, Vera [Hrsg.]: Schülerauslese, schulische Beurteilung und Schülertests 1880-1980. Bad Heilbrunn : Verlag Julius Klinkhardt 2021, S. 47-62. - (Bildungsgeschichte. Forschung - Akzente - Perspektiven)

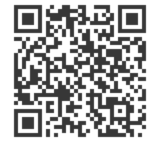

\section{Quellenangabe/ Reference:}

Garz, Jona: "Schriftproben von schwachsinnigen resp. idiotischen Kindern". Testwissen zwischen Psychiatrie und Pädagogik um 1900 - In: Reh, Sabine [Hrsg.]; Bühler, Patrick [Hrsg.]; Hofmann, Michèle [Hrsg.]; Moser, Vera [Hrsg.]: Schülerauslese, schulische Beurteilung und Schülertests 1880-1980. Bad Heilbrunn : Verlag Julius Klinkhardt 2021, S. 47-62 - URN: urn:nbn:de:0111-pedocs-222690 - DOI: 10.25656/01:22269

https://nbn-resolving.org/urn:nbn:de:0111-pedocs-222690

https://doi.org/10.25656/01:22269

in Kooperation mit / in cooperation with:

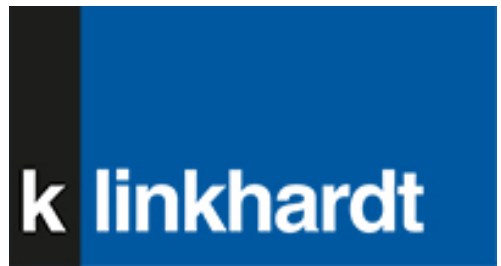

http://www.klinkhardt.de

\section{Nutzungsbedingungen}

Dieses Dokument steht unter folgender Creative Commons-Lizenz: http://creativecommons.org/licenses/by-nc-sa/4.0/deed.de - Sie dürfen das Werk bzw. den Inhalt unter folgenden Bedingungen vervielfältigen, verbreiten und öffentlich zugänglich machen sowie Abwandlungen und Bearbeitungen des Werkes bzw. Inhaltes anfertigen: Sie müssen den Namen des Autors/Rechteinhabers in der von ihm festgelegten Weise nennen. Dieses Werk bzw, der Inhalt darf nicht für kommerzielle Zwecke verwendet werden. Die neu entstandenen Werke bzw. Inhalte dürfen nur unter Verwendung von Lizenzbedingungen weitergegeben werden, die mit denen dieses Lizenzvertrages identisch oder vergleichbar sind.

Mit der Verwendung dieses Dokuments erkennen Sie die Nutzungsbedingungen an.

\section{Terms of use}

This document is published under following Creative Commons-License: http://creativecommons.org/licenses/by-nc-sa/4.0/deed.en - You may copy, distribute and transmit, adapt or exhibit the work in the public and alter, transform or change this work as long as you attribute the work in the manner specified by the author or licensor. You are not allowed to make commercial use of the work. If you alter, transform, or change this work in any way, you may distribute the resulting work only under this or a comparable license.

By using this particular document, you accept the above-stated conditions of use.

\section{Kontakt / Contact:}

peDOcs

DIPF | Leibniz-Institut für Bildungsforschung und Bildungsinformation

Informationszentrum (IZ) Bildung

E-Mail: pedocs@dipf.de

Internet: www.pedocs.de

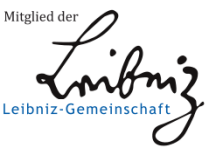


Bildungsgeschichte.

Forschung - Akzente - Perspektiven

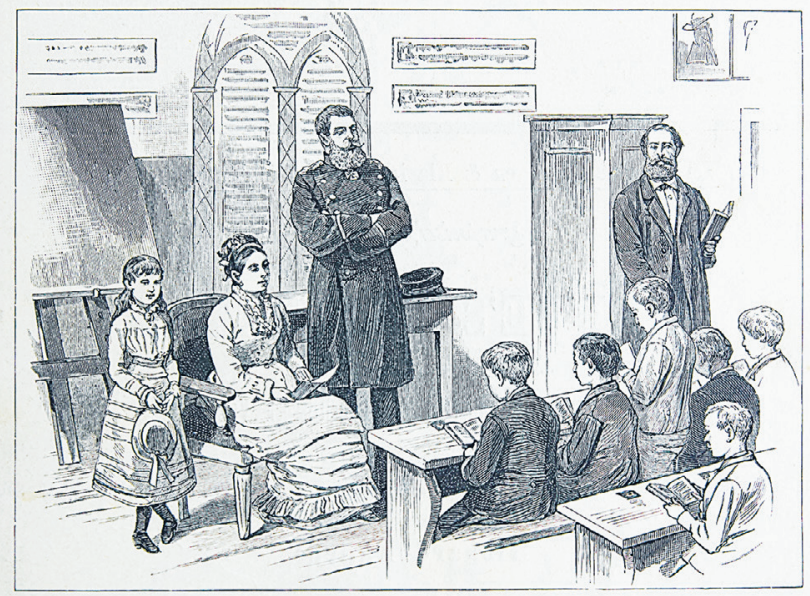

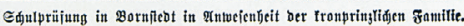

Sabine Reh / Patrick Bühler Michèle Hofmann/Vera Moser (Hrsg.)

\section{Schülerauslese,} schulische Beurteilung und Schülertests 1880-1980 


\section{Sabine Reh \\ Patrick Bühler \\ Michèle Hofmann \\ Vera Moser \\ (Hrsg.)}

\section{Schülerauslese, schulische Beurteilung und Schülertests 1880-1980}


Dieser Titel wurde in das Programm des Verlages mittels eines Peer-Review-Verfahrens aufgenommen. Für weitere Informationen siehe www.klinkhardt.de.

Bibliografische Information der Deutschen Nationalbibliothek Die Deutsche Nationalbibliothek verzeichnet diese Publikation in der Deutschen Nationalbibliografie; detaillierte bibliografische Daten sind im Internet abrufbar über http://dnb.d-nb.de.

2021.1. (C) by Julius Klinkhardt.

Abbildung Umschlag: Schulprüfung in Bornstedt, Quellenangabe: Daheim. Ein deutsches Familienblatt mit Illustrationen 19 (1883) 3, Beil. 2.

Druck und Bindung: AZ Druck und Datentechnik, Kempten.

Printed in Germany 2021.

Gedruckt auf chlorfrei gebleichtem alterungsbeständigem Papier.

Die Publikation (mit Ausnahme aller Fotos, Grafiken und Abbildungen) ist veröffentlicht unter der Creative Commons-Lizenz: CC BY-NC-SA 4.0 International https://creativecommons.org/licenses/by-nc-sa/4.0/ 


\section{Inhaltsverzeichnis}

Sabine Reh, Patrick Bühler, Michèle Hofmann und Vera Moser

Einleitung

Prüfen, Testen, Auslesen und Zuweisen. Zum Inklusions-Paradox

des Schulsystems

Jona Garz, Vera Moser und Stefan Wünsch

Die „Kielhorn-Rede“: Ursprungsmythos der deutschen Hilfsschule

Jona Garz

„Schriftproben von schwachsinnigen resp. idiotischen Kindern“.

Testwissen zwischen Psychiatrie und Pädagogik um 1900

\section{Michèle Hofmann}

Grenzziehungen - Praktiken der Kategorisierung geistig

„anormaler“ Kinder um 1900 in der Schweiz

\section{Patrick Bühler}

„Komplett pessimistisch eingestellt“. Hilfe und Heilung in der

Schweizer Sonderpädagogik zu Beginn des 20. Jahrhunderts

Cristina Alarcón López

Genealogie des Grundschulgutachtens im Zeichen des Dispositivs der

„Schülerauslese“

\section{Rebecca Heinemann}

Im „Mittelpunkt sowohl der theoretisch-psychologischen wie der angewandt psychologischen Arbeit“. Das personalistische Begabungskonzept William Sterns

Susanne Schregel

„Übernormalen-Pädagogik“ und „Begabtenschulen“ zwischen

Kaiserreich und Weimarer Republik

\section{Joachim Scholz}

„In zweifelhaften Fällen mag der Geist der Milde den Ausschlag geben“-

Korrektur und Benotung des deutschen Abituraufsatzes in historischen

Debatten und Praktiken 
Kerrin v. Engelhardt

„Der papierene Drache“ - Der Reifeprüfungsaufsatz zwischen

1890 und 1930

Thomas Hoffmann

Übungsschulen für „Gehirnkrüppel“: Diagnostik, Therapie und heilpädagogische Behandlung hirnverletzter Soldaten 1914-1918

\section{Johanna Lerch}

„Das Kind vor verfehlter Wahl geschützt“. Die Einführung eines „berufspsychologischen Schülerbeobachtungsbogens" in Berliner Schulen, 1917-1923

\section{Fanny Isensee}

„Intelligence tests were given in order to obtain a basis for classifying the pupils" - Die Reclassification Projects in New York City in den 1920er Jahren

Nadja Wenger

„Ihr gebt mich fort, weil ihr mich nicht gern habt." Gutachten der St. Galler Fürsorgestelle für Anormale in den 1940er-Jahren

Michaela Vogt

Das Hilfsschulaufnahmeverfahren als „Grenzzone“ der

Schülerauslese in BRD und DDR

Autor*innenangaben 
Jona Garz

\section{„Schriftproben von schwachsinnigen resp. idiotischen Kindern". \\ Testwissen zwischen Psychiatrie und Pädagogik um 1900}

\section{Idiotie, Schwachsinn und das Problem der Kategorisierung}

„Vom ärztlichen Standpunkt aus, gibt es nur blödsinnige und schwachsinnige Kinder, während der Pädagoge hier einen Unterschied macht; er trennt von den schwachsinnigen Kindern die schwachbefähigten (minderbegabten) und zwar mit demselben Rechte mit welcher er von den blödsinnigen diejenigen, welche an der Grenze des Schwachsinnes stehen, trennt und die zu den bildungsfähigen bringt. Die Schwachbefähigten werden die Gruppe des Schwachsinns ausmachen, welche an das Normale grenzt. Die Grenze nun zu ziehen zwischen schwachbefähigt und schwachsinnig ist sehr schwer“ (Piper 1893a, 4).

Als Hermann Piper (1856-1943) diese Zeilen schrieb, war er bereits mehr als zehn Jahre Leiter der Idiotenanstalt der Stadt Berlin. Sowohl er als auch der wissenschaftliche Diskurs der Zeit hatten sich einer klaren Bestimmung dessen, was als „Idiotie“ bezeichnet wurde, lediglich angenähert: Disziplinen wie Psychiatrie, und Neurologie waren neben der Pädagogik an der Hervorbringung des verfügbaren Wissens beteiligt. Es wurden „blödsinnige“ Kinder identifiziert, die von „schwachsinnigen“, „schwachbefähigten“ und „normalen“ Kindern jeweils zu unterscheiden waren, wobei die Grenze mindestens zwischen „schwachbefähigt“ und „schwachsinnig“ uneindeutig blieb. Ende des 19. Jahrhunderts war die von Erziehungsinspektor Piper vorgetragene Einteilung der „Idiotie“ ein Versuch neu entstehendes Wissen zu kategorisieren und mittels neuer Begrifflichkeiten zu fixieren. Die Hervorbringung von „Idiotie“, im Folgenden verstanden als kulturell verfertigtes Objekt, war begleitet von großen Schwierigkeiten in Bezug auf Uneindeutigkeit und Unabschließbarkeit von Begriffen und Kategorien. So kursierten Ende des 19. Jahrhunderts neben „Idiotie“ Oberbegriffe wie „psychische

1 Dieser Text entstand im Rahmen des von der DFG geförderten Projekts „Profession und normative Ordnungen in der Entstehung der urbanen Hilfsschule: Die Modernisierung der Regierung des Sozialen“, Laufzeit 2017-2020. Ich danke Vera Moser und Stefan Wünsch für ihre Anmerkungen und Hinweise. 
Entwicklungshemmungen“ (Krafft-Ebing 1888, 695; Kraepelin 1904b, 842), „angeborene Defektpsychosen“ (Ziehen 1894, 399) oder "geistig Abnorme“ (Hintz 1897, 783). Für eine weitere Klassifizierung wurden Abstufungen wie „Schwachsinn“, „Blödsinn“ und „Kretinismus“ (Sengelmann 1885, 11-21) oder „Idiotie“, „Imbezillität“ und „Debilität“ (Ziehen 1894, 399-407) und „Vollidioten“, „Halbidioten“ und „Schwachsinnige“ (Gündel 1896, 89-91) vorgeschlagen. In Bezug auf die Vielzahl von kursierenden Begriffen und Grenzziehungen, die nebeneinander kursierten, wurde bereits um 1900 in Veröffentlichungen Unverständnis geäußert (vgl. Falch 1880/81, 99) und kritisiert, dass nicht einmal „zwei Autoren in ihren Begriffen überein[treffen]" (Gündel 1896, 74). Doch nicht nur auf der begrifflichen Ebene tummelten sich die Definitionen, die „Ätiologie der Idiotie“ (Piper 1893b) war ebenso umstritten wie ihre Klassifizierung. So wurden neben einer bereits abgelaufenen bzw. chronifizierten Hirnerkrankung hereditäre Faktoren wie Vererbung, Alkoholismus eines Elternteils, diverse Erkrankungen der Mutter während der Schwangerschaft sowie allgemein Fehlentwicklungen der Großhirnrinde diskutiert (vgl. ebd.). Übereinstimmung herrschte Ende des 19. Jahrhunderts lediglich darüber, dass „Idiotie“ bzw. „Schwachsinn“ nicht heilbar waren (vgl. Kraepelin 1904b, 854-858). Hinsichtlich der Klassifizierung war man sich uneins, ob Sprachvermögen (Esquirol), Aufmerksamkeit (Sollier) oder Auffassungsvermögen (Emminghaus) am adäquatesten die Einteilung der „Idioten“ ermöglichte (vgl. Piper 1897, 789-790). Das Wissensobjekt „Idiotie“ präsentierte sich am Ende des 19. Jahrhundert als eines mit unscharfen Begriffen und Grenzziehungen, wobei sowohl die Frage, auf welches Phänomen sich diese bezogen, als auch die Grenze hin zur Normalität umstritten waren (vgl. Krafft-Ebing 1888, 703; Gündel 1896, 74; Hintz 1897, 821; Ziehen 1894, 409).

Die Ausdehnung der Psychiatrie, also die Vergrößerung des reklamierten Zuständigkeitsbereichs dieser neuen medizinische Disziplin, führte zu immer neuen Diagnosen, zu der auch „Idiotie“ und „Schwachsinn“ gehörten (vgl. Bühler 2017). Die Tatsache, dass diese Erkrankungen schnell als unheilbar galten, eröffnete der Pädagogik die Möglichkeit, sich an der Behandlung ,,idiotischer" Kinder zu beteiligen. In diesem Wissensfeld waren sowohl Psychiater als auch Pädagog*innen an der Hervorbringung von „Idiotie“-Wissen beteiligt. Zentral hierfür war es, Wege zu (er-)finden, dem Problem, dass der Ort, an dem der Sitz der „Idiotie“ vermutet wurde - im Kopf -, nicht einsehbar war, zu begegnen.

Um einen Versuch der Sichtbarmachung von Gehirnfunktionen, und zwar mittels sogenannter Schriftproben geht es im Folgenden. Zunächst wird ein kurzer Überblick zur Geschichte der Produktion von Wissen in der Psychiatrie gegeben. Hierbei liegt der Fokus auf der Entwicklung der Disziplin Psychiatrie im 19. Jahrhundert im deutschsprachigen Raum, die insbesondere mit der Anerkennung als empirische Wissenschaft und dem Problem der Sichtbarmachung von pathologischen Zuständen des Gehirns rang (2). Im Anschluss wird eine der um 
die Jahrhundertwende diskutierten Lösungen dieses Problems vorgestellt und auf ihre epistemologischen Effekte hin näher beleuchtet. Als Beispiel dienen hierfür Schriftproben von „schwachsinnigen Kindern“, die der Erziehungsinspektor der Idiotenanstalt Dalldorf bei Berlin, Hermann Piper, Ende des 19. Jahrhunderts erhob und auswertete (3). Den Abschluss bildet ein knapper Ausblick darauf, wie Hilfsschulen und Hilfsschullehrer*innen sich von der Möglichkeit der Generierung von Testwissen abwandten und sich auf ihre Kompetenz der pädagogischen Beobachtung zurückzogen (4).

\section{Psychiatrie, Gehirnfunktionen und das Problem der (Un-)sichtbarkeit}

„Idiotie“ ist eine Diagnose, deren Herkunft sich in der Medizin bzw. Psychiatrie des späten 18. und frühen 19. Jahrhunderts verorten lässt (vgl. Gstach 2015). Bis ins späte 19. Jahrhundert hinein wurde „Idiotie“ als eine Schwächung sämtlicher Seelenvermögen gedeutet. Die Seele wurde als immaterielle Substanz verstanden, die Lebensursache allen menschlichen Lebens war und somit auch die Voraussetzung für sinnliche Wahrnehmung und Denken. Dies begann sich mit dem Auftreten von Franz Joseph Galls Theorie, dass das Gehirn den materiellen Sitz aller geistigen Vermögen darstellt, langsam zu ändern (vgl. Canguilhem 1989, 9-10; Hagner 2000, 89-137). Die Lehre von der Lokalisation der geistigen Eigenschaften ermöglichte langfristig eine Entwicklung, in deren Lauf allmählich die Vorstellung einer Seele als Sitz des geistigen Vermögens verschwand. Stattdessen wurde das Gehirn sowohl zum Sitz des geistigen Vermögens als auch zu einem Organ unter anderen. Die These der Lokalisierbarkeit bestimmter Funktionen im Gehirn wurde im Laufe des 19. Jahrhunderts zentral für die sich herausbildende Psychiatrie. Insbesondere im Verbund mit der Physiologie entwickelte sich die Psychopathologie, der es mit Experimenten zunächst gelang, den Sitz der Sprachfunktionen im Frontalhirn zu lokalisieren und die Ende des 19. Jahrhunderts sowohl innerhalb der Wissenschaft als auch im Populärwissen zunehmend an Einfluss gewann (vgl. Canguilhem 1989). In der Folge wurde das Gehirn (und nicht die Seele) das, was den Menschen zu dem machte, was er ist.

Der Lokalisationsgedanke wurde für die Psychiatrie als Wissenschaft zu einem Problem. Denn das Gehirn, auf das nun sämtliche menschliche Äußerungen zurückgeführt wurden, war von außen nicht einsehbar, sämtliche psychische Erkrankungen wurden aber auf eine Störung der Funktionen dieses Organs zurückgeführt. Die Psychiatrie, wollte sie ihrem Versprechen der Diagnose und Heilung psychisch Kranker gerecht werden, musste sich somit Techniken bedienen bzw. hervorbringen, die die Sichtbarmachung ihres Gegenstandes ermöglich- 
ten. Dies geschah zunächst über das Beobachten, Erkennen, Aufschreiben und Einordnen von Symptomen. In der Medizin etablierte sich seit dem 18. Jahrhundert eine Praxis des Aufschreibens, die wohl zunächst begründet war in der Abrechnungsökonomie der Verwaltung der Krankenhäuser (vgl. Hess 2010), sich aber im Vollzug zu einer Technik der Wissensproduktion entwickelte (vgl. Borck \& Schäfer 2015). Die Ärzte waren angehalten, möglichst genau und möglichst alle Beobachtungen am Krankenbett in einer Krankenakte zu notieren. Da das in der Realität nicht möglich war, wurden Nachvollziehbarkeit und Reduktion zu den zentralen Aspekten einer gut geführten Krankenakte, die gleichsam immer unter dem Verdacht stand, nicht ausreichend objektiv verfasst worden zu sein (vgl. Ledebur 2011). Trotzdem stellten die Akten die einzige Möglichkeit dar, Daten überhaupt jederzeit Zeit abzurufen. Zudem hielten sie den Ort bereit, an dem die Beobachtungen der flüchtigen Ausdrucksbewegungen sich in Form der Schrift der Psychiater zuallererst materialisierten (vgl. Ledebur 2015). Die Wissensproduktion bestand in der Folge insbesondere darin, die Krankenakten verschiedener Fälle nebeneinanderzustellen und ähnliche Fälle zu Ausgangsbeispielen zu gruppieren. Diese aus der Materialsammlung der Krankenakten gewonnenen Beispiele bildeten die empirische Grundlage für theoretische Generalisierungsversuche, die in (neuen) Diagnosen mündeten (vgl. Engstrom 2005).

So entstand durch Beobachten, Aufschreiben und Gruppieren der Ausdrucksformen ein immer breiterer und feingliedriger Diagnosen-Katalog. Die Diagnosen wurden wiederum vergeben, indem die Ärzte weiterhin sämtliche Ausdrucksformen, also Verhaltensweisen, die beobachtbar waren und mittels derer man auf den inneren Zustand der*des Patient*in schließen konnte, aufzeichneten und mit bestehenden Gruppierungen abglichen. Im Zuge von Diagnostizierung und Behandlung wurden so quasi endlos neues Wissen und neue Diagnosen produziert, die immer mehr Formen von Verhalten abdeckten und pathologisierten (vgl. Bühler 2017). In Preußen führte die Gemengelage aus Ausdehnung der Zuständigkeit der Psychiatrie, Bevölkerungsexplosion und Verstädterung zwischen 1877 und 1901 zu einer Verdoppelung der Anstaltspsychiatrien bei gleichzeitiger Verdreifachung der Anzahl der Insassen. Die Psychiatrien waren dadurch häufig überbelegt (vgl. Engstrom 2003, 30-31). Um die Erwartungen der sie finanzierenden kommunalen Verwaltungen zu erfüllen, mussten die Psychiater in der Lage sein, zuverlässige Diagnosen, Therapien und Prognosen zu erstellen, denn die Kranken sollten schnellstmöglich geheilt oder zumindest gebessert entlassen werden (vgl. Borck \& Schäfer 2015, 14). Diesem Anspruch gerecht werden sollte die sich Ende des 19. Jahrhunderts etablierende wissenschaftliche Psychiatrie, die nicht mehr ausschließlich auf kleinteiliger Beobachtung und der Verschriftlichung der flüchtigen Ausdrucksformen (verstanden als jegliche Form von sprachlicher wie auch nicht-sprachlicher Äußerung) beruhen sollte (vgl. Ledebur 2015). Stattdessen wurde eine objektive, naturwissenschaftliche Psychiatrie entworfen, die auf 
drei Säulen beruhen sollte: erstens (Neuro-)Pathologie, zweitens systematisierte klinische Untersuchungsmethoden und drittens Übernahme von Methoden aus der Physiologie und Psychologie (Borck \& Schäfer 2015, 10). Die Übernahme der Methoden bedeutete vor allem den Eintritt in die „Kultur des Experiments“ (Rheinberger 2007, 135-138). Anders als in der klinischen Beobachtung, deren Fokus auf der Einzigartigkeit der Phänomene und Menschen liegt und die sich immer bemühen muss vom Besonderen zum Allgemeinen zu kommen, geht experimentelle Wissenschaft umgekehrt vor. Ihre Aufmerksamkeit ist auf die Allgemeinheit der Phänomene und somit auf ihre Reproduzierbarkeit gerichtet (vgl. Hagner 2000, 23). Der Einsatz von Experimenten, der insbesondere in der experimentellen Psychologie florierte und der in Teilen der Psychiatrie an Einfluss gewann, lässt sich gleichfalls als Versuch verstehen, dem Problem der ungenauen Beobachtung und Diagnostik beizukommen (vgl. u.a. Ankele 2012; Ledebur 2013). Ziel und Anspruch war es neues, objektives und zugleich überprüfbares Wissen zu produzieren, das gleichzeitig die ersehnte Eindeutigkeit herstellen sollte (Borck \& Schäfer 2015, 11-12).

\section{Testwissen aus Schriftproben als Lösung?}

Im Kontext einer psychiatrischen Wissenschaft entstanden um 1900 eine Vielzahl von Experimenten, mittels derer etwas über den Zustand des Gehirns ausgesagt werden können sollte. Dazu gehören u.a. Experimente, in denen Veränderungen des Pulses festgestellt, psychische Zeitmessungen, bspw. der Geschwindigkeit mentaler Prozesse, vorgenommen, chronoskopische Vermessungen veranlasst, Vorstellungsverbindungen untersucht und Gemütsmessungen mittels eines sogenannten Zitterapparates geprüft wurden (vgl. Kraepelin 1904a, 347-369). In dieses weite Feld der Experimente reihen sich unterschiedliche Varianten von Schriftproben ein. Im 17. Jahrhundert hatte sich in der Graphologie die Gewissheit entwickelt, dass es möglich sei, über Schriftstücke Rückschlüsse auf die Persönlichkeit der Schreibenden zu ziehen. Die darin angelegte Vorstellung, dass sich unsichtbare Vorgänge des Körpers auf das Schriftbild übertrugen, sorgte dafür, dass Schreiben als Diagnoseinstrument auf ein gesteigertes Interesse in einigen Bereichen der psychiatrischen Wissenschaft des 19. Jahrhundert stieß (vgl. Schäfer 2005, 242-244; Kammer 2013, 226-233). Patient*innen wurden aufgefordert in unterschiedlichen Situationen zu schreiben und zu zeichnen, per Diktat oder aus dem Gedächtnis, wobei in aller Regel die Form und Anordnung der Buchstaben auf dem Blatt und nicht der Inhalt interessierte (vgl. Köster 1904). Die Psychiater, bis eben noch selbst am Schreiben, konnten nun dazu übergehen die Schrift ihrer Klientel zu interpretieren. So entstand in der Psychiatrie ein „Schrift- 
Wissen“ (Kammer 2013), das, anders als die Graphologie, nicht mehr meinte, Charaktereigenschaften im Schriftbild zu erkennen, sondern die Handschrift als materialisierter Ausdruck der Störungen des Gehirns verstand. Insbesondere die Möglichkeit, durch die Auswertung von Schriftbildern und Zeichnungen von Patient*innen der Flüchtigkeit von Ausdrucksbewegungen entgegenzuwirken, schien vielversprechend und regte zu zahlreichen Untersuchungen an (vgl. u.a. ebd.; Schäfer 2005; Wittmann 2005). Zudem konnte „über das Papier als Trägermedium die Objektivität dieser Ausdrucksbewegungen materiellen Rückhalt gewinnen" (Ankele 2012, 98).

Der eingangs zitierte Erziehungsinspektor Hermann Piper war einer derjenigen, die sich auf dem Gebiet der Schreibexperimente versuchten. 1893 veröffentlichte er die Ergebnisse seiner Versuche unter dem Titel Schriftproben von schwachsinnigen resp. idiotischen Kindern, gesammelt und zusammengestellt von Hermann Piper. Er war 1881 als examinierter Gemeindeschullehrer zum ersten Erziehungsinspektor der neu gegründeten Städtischen Idiotenanstalt zu Dalldorf bei Berlin ernannt worden. Deren Bau war im März 1880 von der Stadtverordnetenversammlung beschlossen worden: Auf dem Gelände der Städtischen Irrenanstalt sollte ein separates Gebäude entstehen, in dem 100 ,idiotische“ Kinder untergebracht und beschult werden konnten (vgl. Magistrat der Stadt Berlin 1880). Die Idiotenanstalt war der Städtischen Irrenanstalt verwaltungstechnisch unterstellt und stellte eine Art Internatsschule dar (vgl. Ideler \& Magistrat der Stadt Berlin 1883). Die Kinder wurden auf Antrag der Eltern, bestätigt durch ein Attest des Armenarztes, durch eine Entscheidung des Kuratoriums der Anstalt aufgenommen. Sie konnten auf Antrag der Angehörigen entlassen werden, Urlaub machen und an den Wochenenden Besuch empfangen (vgl. Magistrat der Stadt Berlin \& Deputation für die Städtische Irrenpflege 1899). Vormittags und nachmittags erhielten die Kinder Unterricht, je nach Alter bis zu 38 Wochenstunden, und waren jenseits des Unterrichts in anfallende Arbeiten eingebunden (vgl. Piper 1883).

Inwieweit Pipers Arbeitsumfeld ausschlaggebend für sein Interesse an medizinischen Experimenten war, ist nicht zu klären, der ausgebildete Lehrer zeigte sich in jedem Fall offen für die Methoden der Ärzte aus den Nachbargebäuden. ${ }^{2}$ Piper stieß dabei auf eine Studie des Breslauer Arztes Prof. Dr. O. Soltmann. Dieser hatte im Rahmen einer klinischen Studie zu Schrift und Spiegelschrift bei Gesunden und Kranken Kindern geforscht und veröffentlicht (vgl. Goldscheider 1891). Soltmann hatte in seinem Experiment „gesunde“ und „idiotische“ Kinder sowohl mit

2 Das zeigt sich nicht nur an der Auseinandersetzung mit der Methode der Schriftproben und Kontakten mit bekannten Kinderärzten, Physiologen und Sprachheilärzten (vgl. Opitz 1952, 97), sondern auch an Veröffentlichungen wie Die Ätiologie der Idiotie (Piper 1893b), Die Sprachgebrechen bei idiotischen Kindern und ihre Heilung (Piper 1891) oder Statistik über die Sprachgebrechen der in den nachfolgenden Anstalten (Internaten) bezw. Schulen (Externaten) befindlichen schwachsinnigen Kinder (Piper 1895). 
der rechten als auch mit der linken Hand schreiben lassen. Diese Schriftproben verglich er untereinander und stellte fest, dass er anhand von mit der linken Hand geschriebener Spiegelschrift Rückschlüsse auf den geistigen Zustand der Kinder ziehen konnte. Von 18 untersuchten „idiotischen“ Kindern hatten 16 mit der linken Hand spiegelverkehrt geschrieben, während es unter den „normalen“ „nur wenige“ waren, die aber "durchweg nicht ganz gesund“ waren (ebd., 414). Störungen der Funktion der Großhirnrinde und infolgedessen die Unfähigkeit des Gehirns, einen Abgleich der Schrift mit dem zentral abgelegten Erinnerungsbild zu bewerkstelligen, führten laut Soltmann dazu, dass „idiotische“ Kinder, wenn sie aufgefordert wurden mit der linken Hand zu schreiben, Spiegelschrift schrieben. So kam er zu dem vielzitierten Schluss, dass „wir in der Spiegelschrift den Spiegel einer kranken Seele [!] erblicken“ (zit. nach ebd., 415).

Wie oben bereits zitiert, stand auch Piper vor Schwierigkeiten der Abgrenzung der verschiedenen Grade von „Idiotie“ bzw. „Schwachsinn“ (vgl. Piper 1893a, 4), weshalb eine Methode, die ermöglichte, schnell und zuverlässig den mentalen Zustand eines Kindes zu bestimmen, wohl vielversprechend erschien. Hinzu kam, dass Piper es einfacher fand, „Blödsinn“ und verschiedene Formen des „Schwachsinns" in der Theorie zu unterscheiden, als dies in der Praxis zu tun - weshalb er in seinem Alltag in der Regel davon absah und stattdessen grob „bildungsfähige“ von „bildungsunfähigen“ Kindern unterschied. In der ersten Gruppe der „Bildungsfähigen“ fasste er "gewöhnungsfähige Blödsinnige“, „Schwachsinnige“, „Schwachbefähigte“, „schwachsinnige Epileptiker“ und „schwachbefähigte Epileptiker“ zusammen, die zweite Gruppe bildeten die „bildungsunfähigen Blödsinnigen“. Abgesehen von den "Schwachbefähigten“ (die in die Normalschule gehörten) seien hiermit, so Piper, „die Insassen von Erziehungsanstalten für Schwachsinnige resp. Idioten und für Epileptiker gekennzeichnet" (ebd., 5). Er konnte also zum Zwecke der Bildung grob unterscheiden, wer den Unterricht der Idiotenanstalt besuchen sollte, eine weitere Differenzierung war ihm, zumindest innerhalb kurzer Zeit, nicht möglich. In der Erwartung, „ein wertvolles Mittel, resp. ein beachtenswerten Maßstab zur Beurteilung des intellektuellen Zustandes unserer geistig schwachen Zöglinge“ (ebd., 7) zur Hand zu haben, wiederholte Piper im Jahr 1892 den Versuch, anhand von Schriftbildern den Schweregrad der "Idiotie“ festzustellen und war somit einer derjenigen, die zur Hervorbringung des Objekts „Idiotie" beitrugen.

In der Idiotenanstalt Dalldorf waren zur Zeit der Durchführung der Schriftproben 237 Zöglinge untergebracht, von denen täglich 202 in vier aufsteigenden Klassen unterrichtet wurden. Schreibunterricht wurde erst ab der 5. Klasse erteilt, weshalb Piper von diesen Kindern keine Schriftproben abnahm. Von den restlichen 149 Kindern gaben alle eine Schriftprobe ab (vgl. ebd.). 
Piper beschreibt den Versuchsaufbau wie folgt:

„Die Schriftproben mit der linken und darnach rechten Hand wurden nicht in den Klassenzimmern, wo bei derartigen Gelegenheiten ein Kind das andere genau beobachtet und bald merkt, um was es sich handelt, vorgenommen, sondern es wurden im Verlauf von einigen Wochen nach und nach ohne Aufsehen die Kinder einzeln in ein Zimmer gebracht, um hier die Schriftprobe auszuführen. [...] Jedes Kind wurde absichtlich daran erinnert, dass wir stets mit der rechten Hand schreiben, diesmal aber einen Versuch mit der linken Hand machen wollen. [...] Die Schüler der I. bis incl. III. Klasse schrieben nach Diktat Ziffern, einzelne Buchstaben, Namen während die der IV. und V. Klassen mit Rücksicht auf ihren intellektuellen Zustand nur einzelne Buchstaben schreiben konnten“" (ebd., 7).

Er nahm die Angelegenheit im Sinne eines Experiments ernst, versuchte gleiche Bedingungen für alle zu schaffen und Störfaktoren, die die Korrektheit bzw. Objektivität der Beobachtungen beeinträchtigt hätten, auszuschließen. Zudem wählte Piper je nach Lernstand unterschiedliche Aufgaben.

In veröffentlichter Form sehen die Schriftproben folgendermaßen aus (siehe Abb. 1 und Abb. 2). Sie sind zunächst absteigend geordnet nach Klasse und dann danach, ob mit der linken Hand Spiegelschrift geschrieben wurde oder nicht. Sowohl die Schriftproben der linken als auch der rechten Hand sind je Kind abgedruckt; die diktierten Zahlen und Buchstaben sind jeweils identisch. Zudem sind neben dem Alter des Kindes die anderweitig gewonnene Diagnose („schwachsinnig“, „fast blödsinnig“, „blödsinnig“) sowie Bemerkungen zu Gemüt („gutmütig“, „zänkisch“, „treibt gern Alotria“, „exaltiert") und physiologische Auffälligkeiten („geht stets im Kiebitzlauf“, „Verbildung am Gaumen“, „zwergartig“) vermerkt. Piper wertete die Schriftproben statistisch aus, jede Klasse wurde einzeln aufgeschlüsselt, vorhandene Epilepsie wurde ebenso vermerkt wie die Qualität des Schriftbilds der rechten Hand. Über alle Klassen hinweg schrieben $47 \%$ der Zöglinge mit links Spiegelschrift, ${ }^{3}$ wobei die Zahl in den unteren Klassen deutlich zunimmt und bei denjenigen mit epileptischen Störungen seltener vorkommt. Pipers (1893a, 16) Fazit lautet, dass erstens Kinder mit „centralen Störungen“ häufiger Spiegelschrift schrieben, aber zweitens umgekehrt nicht alle Kinder mit „centralen Störungen“ Spiegelschrift schrieben. Und „3. Die Spiegelschrift wird uns neben anderen Erscheinungen bei der Aufstellung der Prognose ein wertvolles Mittel resp. ein beachtenswerter Massstab zur Beurteilung des intellektuellen Zustandes unserer geistig schwachen Zöglinge“.

3 Piper hatte keine Kontrollgruppe; aber sowohl Soltmann als auch später Goldscheider gingen davon aus, dass Spiegelschrift bei „normalen“ Kindern nicht vorkam. 


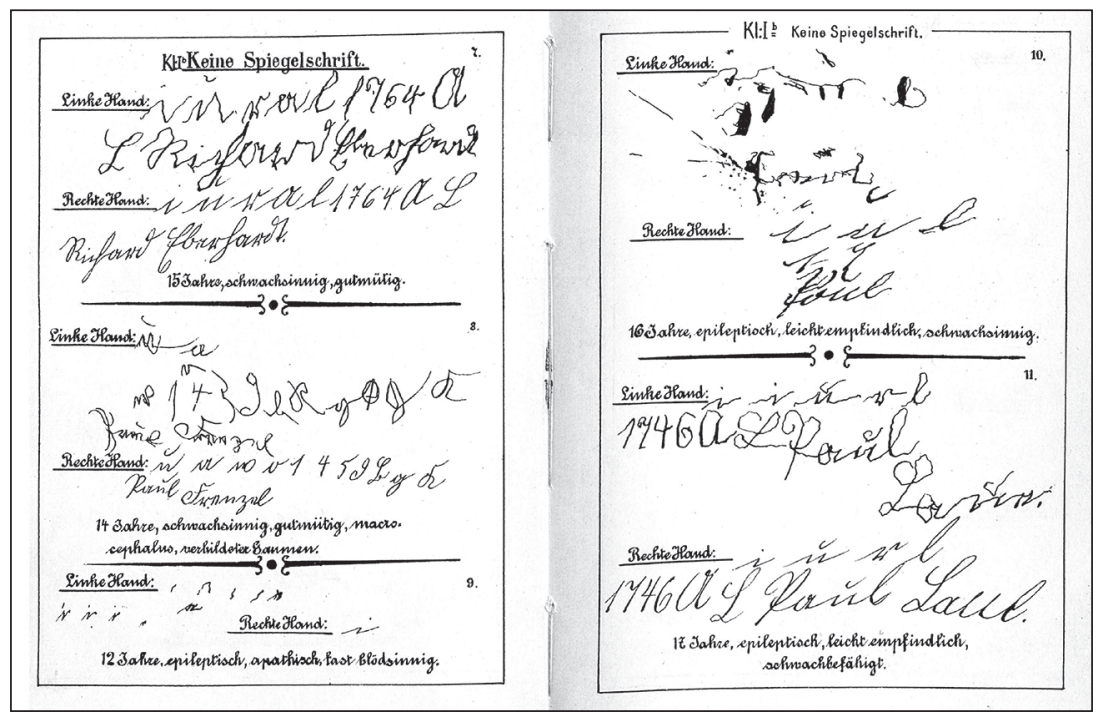

Abb. 1: Seite aus Piper (1893a).

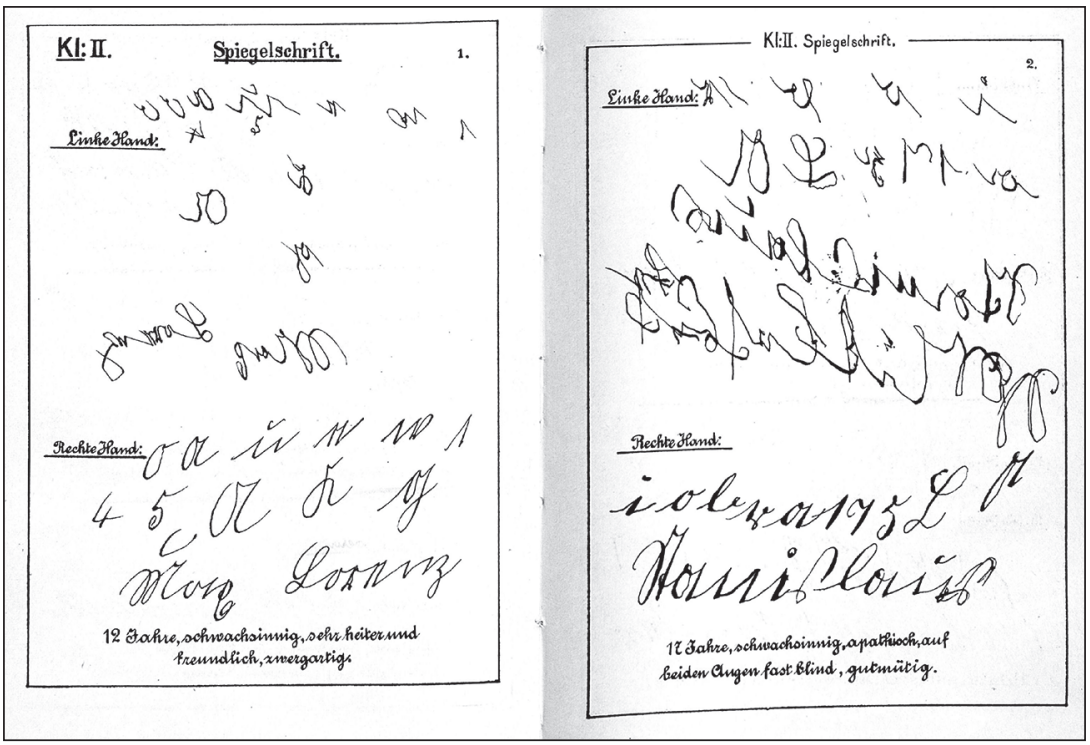

Abb. 2: Seite aus Piper (1893a). 
Folgendes ist meines Erachtens an Pipers Experiment und der anschließenden Veröffentlichung bemerkenswert: Sein eigentliches Ansinnen, ein Instrument zur Beurteilung des geistigen Zustandes seiner Zöglinge zu erlangen, wird de facto nicht erreicht. Die erzielten Ergebnisse sind dafür nicht eindeutig genug, da weniger als die Hälfte der „schwachsinnigen“ Kinder Spiegelschrift schrieben. Die Veröffentlichung des Experiments ermöglichte es dem Erziehungsinspektor aber Teil des medizinisch-wissenschaftlichen Diskurses der Zeit zu werden. So wurde Piper bspw. von Emil Kraepelin (1904b, 864), einem der einflussreichsten Psychiater um 1900, zitiert. Piper beteiligte sich damit folglich an jenem „Schrift-Wissen“ der Psychiatrie, das der Handschrift ein „privilegiertes symptomatisches Potenzial“ zuschreibt (Kammer 2013, 228). Neben Soltmann führten u.a. Leopold Treitel (1893) und ein Lehrer namens E. Kannengießer (1895) Pipers Experiment durch - mit ähnlich uneindeutigen Ergebnissen. Im Vergleich zu den beteiligten Ärzten fällt auf, dass Piper sich im Gegensatz zu diesen nicht für die Theorien interessierte, die hinter der Überzeugung standen, Spiegelschrift sage etwas über die zentralen Störungen der Großhirnrinde aus. Die Veröffentlichung von Soltmann wurde in der medizinischen Fachpresse durchaus kritisch diskutiert, auch im Hinblick auf die zugrundeliegenden Theorien (vgl. Goldscheider 1891; Ebbinghaus 1897; Lomer 1913). Die physiologisch schwierigen Fragen in Bezug auf ob und wie das Auge oder das Gehirn die Muskeln beim Schreiben kontrollieren und die Zweifel, ob man also beim Untersuchen der Schrift überhaupt direkte Rückschlüsse auf das Gehirn treffen könne, führten dazu, dass zunächst der Stabsarzt Goldscheider (1892) dazu überging, statt der Handschrift selbst den Druck, der beim Schreiben auf das Papier ausgeübt wird, zu untersuchen. Dabei ging es nicht mehr nur darum, „Idiotie“ über das Schriftbild feststellen zu können, sondern die Hoffnung war, jegliche Form der psychiatrischen Auffälligkeit im Schriftbild sichtbar machen zu können. Um dieses Verfahren weiter zu objektivieren und in Zahlenwerte zu übersetzen, erfand Kraepelin (1904a) in der Folge die sogenannte Schriftwaage. Mit ihr konnten Druck- und Bewegungsvorgänge sowie Schreibweg und Schreibzeit aufgezeichnet werden und in eine grafische Darstellung übersetzt werden - die Schrift wurde zu einer Form der Kurve, die sich selbst schreibt. Die damit mögliche Quantifizierung des Schreibens sollte, so Kraepelins (1904a, 357) Hoffnung, dazu beitragen, das "Streben nach genauen zahlenmäßigen Ergebnissen“, wie sie in der naturwissenschaftlich geprägten Medizin erforderlich waren, zu befriedigen (vgl. auch Schäfer 2005).

Am bemerkenswertesten in Bezug auf Pipers Schriftproben erscheint allerdings der Wert, den er seinen eigenen Beobachtungen und Diagnosen beimaß. Letztlich befand er sich - wie die Psychiatrie auch - in einem Dilemma zwischen qualifizierender Beobachtung und quantifizierendem Experiment, zwischen dem Versuch, aus individuellen Beobachtungen Diagnosen und vor allem auch Prognosen zu erstellen, und dem Versuch, aus allgemeinen Erhebungen Rückschlüsse auf den 
einzelnen Fall zu ziehen (vgl. Hagner 2000, 23). Wobei Letzteres Objektivität und vor allem auch Schnelligkeit beim Erstellen von Diagnosen versprach. Piper schien sich dessen bewusst zu sein, führte er doch das Experiment in der Hoffnung durch, ,ein wertvolles Mittel resp. ein[en] beachtenswerte[n] Massstab zur Beurteilung des intellektuellen Zustandes unserer geistig schwachen Zöglinge“ gefunden zu haben (Piper 1893a, 16). Dennoch wurde bei der Veröffentlichung unter jeder der Schriftproben die durch Beobachtung bereits gewonnene Diagnose vermerkt. Offenbar ließ sich Piper in keiner Weise durch gegenteilige Ergebnisse des Experiments verunsichern. Stattdessen verließ er sich nach wie vor auf die Verschriftlichung genauer Beobachtungen zur Hervorbringung von Diagnosen. Sieht man sich die erhaltenen Krankenakten der Idiotenanstalt Dalldorf an, die nach der Durchführung der Schriftproben angelegt wurden, bestätigt sich das Bild. Die Anamnese wurde durch Fragebögen (vgl. Magistrat der Stadt Berlin \& Deputation für die Städtische Irrenpflege 1899), die insbesondere Vorerkrankungen, auch der Eltern, abfragten sowie Fähigkeiten, „Gemütszustand“ und physische Auffälligkeiten erhoben. Diese Fragebögen wurden in der Regel von den Armenärzten nach einem Gespräch mit den Eltern ausgefüllt. In der Anstalt selber wurden die Erziehungsberechtigten nach einer Vorgeschichte befragt, welche in Form eines Fließtextes niedergeschrieben und in die Krankenakten geheftet wurde. In aller Regel reichten diese Informationen zur Vergabe der Diagnose „Idiotie“, die für die Aufnahme in die Anstalt Voraussetzung war. In einigen Akten ist aber zusätzlich eine Schriftprobe enthalten, die das aufzunehmende Kind sowohl mit der der linken als auch der rechen Hand auf ein loses Blatt schrieb (bspw. LAB A Rep. 003-04-04 Nr. 2182; 5668; 5864; 5659; 5861; 5835; 7145). In den Akten wird darauf in keiner Art und Weise Bezug genommen, weder auf die Durchführung noch auf das Ergebnis. Wie es scheint, wurde in Dalldorf Schriftproben bei der Vergabe von Diagnosen und Erstellung von Prognosen auch im weiteren Verlauf des Aufenthalts des Kindes in der Anstalt keine besondere Rolle eingeräumt. Stattdessen wurde dem Befragen, Beobachten und Aufschreiben der Vorzug bei der Hervorbringung von Wissen über das einzelne Kind gegeben wie im Übrigen auch in der Psychiatrie, die nach einer Hochphase um 1900 die Diagnostik mittels Schrift- bzw. Schreibbildern aufgab (vgl. Schäfer 2005, 265). Der Enthusiasmus, mittels Experimenten eine technische Lösung des Problems der Diagnostik psychischer Devianz zu finden und sich dabei gleichsam dem Rationalisierungsdruck der Kommunen zu beugen, hatte Ende des 19. Jahrhunderts zwar zunächst zu einer Explosion an verschiedensten Techniken der Wissensproduktion geführt, von denen viele den Erwartungen jedoch nicht standhielten. Insbesondere in Bezug auf das "Schrift-Wissen“ setzte sich sowohl in der Pädagogik als auch in der Psychiatrie die Überzeugung durch, dass der Versuch, Vorgänge im Gehirn durch die schreibende Hand sichtbar zu machen, weder zuverlässig noch theoretisch herleitbar war. 


\section{Testwissen vs. Beobachtungswissen}

Die Hoffnung, durch Tests zu objektiven und vor allem zu wiederholbaren Erkenntnissen über den Zustand eines menschlichen Gehirns zu gelangen, verschwand dennoch nicht, wie ein Blick auf den Erfolg standardisierter Tests im Schulsystem (vgl. z.B. Linn 2001) und auf die Geschichte der Intelligenztestung im 20. Jahrhundert (vgl. z.B. Carson 2014) zeigt. Zentral für diese weitere Entwicklung waren zwei Wissenschaftler, die sich im Raum zwischen Psychiatrie und Pädagogik bewegten und weiter an Experimenten in Form von Tests arbeiteten, Alfred Binet und Théodore Simon, die bereits 1904 ihre Méthodes nouvelles pour le diagnostic du niveau intellectuel des anormaux vorlegten (vgl. Binet \& Simon 1904). Ziel der beiden war es, eine differentielle Methode zu finden, um den Grad der Intelligenzminderung festzustellen, wie sie ein Jahr später in einem auf Deutsch erschienenen kurzen Artikel in der interdisziplinären Zeitschrift Eos berichten (vgl. Binet \& Simon 1905). Der umfangreiche Test wurde 1910 durch den Psychologen Otto Bobertag $(1910 ; 1911)$ ins Deutsche übertragen. In den folgenden zwei Jahren wurden im deutschsprachigen Raum mehrere größere Sammeltestungen mit dem Binet-Simon-Bobertag-Test durchgeführt und die Ergebnisse veröffentlicht (vgl. Chotzen 1912; Bloch 1915). Im Gegensatz zu den Schriftproben war dieser Test insofern erfolgreicher, als dass sich die Ergebnisse verallgemeinern und reproduzieren ließen - beides zentrale Kriterien der neuen Wissenschaftlichkeit. So wurde der Test zwar in Psychiatrien und Armeen, auch international, standardmäßig eingesetzt, um die intellektuelle Leistungsfähigkeit Einzelner zu beurteilen und Diagnosen wie „Idiotie“ und „Schwachsinn“ zu stellen (vgl. etwa Carson 2014). Dennoch konnte sich der Binet-Simon-Test in Deutschland nicht als Instrument durchsetzen, mittels dem die Zuweisung in die Hilfsschulen erfolgte.

1915, noch während Hermann Piper Erziehungsinspektor in Dalldorf war, wurde das Kind Emil L. in die Idiotenanstalt überwiesen. Emil L. war am 10.02.1908 in Berlin geboren und auf Veranlassung der Armendirektion im Alter von drei Jahren in die Irrenanstalt Dalldorf aufgenommen worden. Nach zweijährigem Aufenthalt wurde noch in der Irrenanstalt "nach Binet-Simon-Bobertag“ getestet, das Intelligenzalter von 3 3/8 Jahren festgestellt und die Verlegung in die Idiotenanstalt veranlasst. Die Aufnahme in die Idiotenanstalt erfolgte durch die üblichen Fragebögen (vgl. LAB A Rep. 003-04-04 Nr. 5648). Die Akte von Emil L. ist von den 11 aus der Irrenanstalt im Zeitraum bis etwa 1920 erhaltenen Patientenakten, die sich auf Kinder beziehen, die Einzige, in der sich Notizen zu einem durchgeführten Binet-Simon-Test finden. Allerdings wurde der Test eben nicht in der Idiotenanstalt, sondern in der Irrenanstalt durch den zuständigen Arzt, vor Vergabe der Diagnose „Idiotie/Imbezillität“ und der Überweisung an die Idiotenanstalt, durchgeführt. In den 34 aus der Idiotenanstalt erhaltenen Patientenverwaltungs- 
akten finden sich keine Notizen zur Durchführung des Tests. Denn der Großteil der Zöglinge, die in der Idiotenanstalt Dalldorf untergebracht wurden, wurden von einer Gemeindeschule und nicht aus der Irrenanstalt heraus überwiesen. In den Gemeindeschulen Berlins wurden diejenigen Kinder, die zwei Jahre ohne Erfolg die unterste Klasse besucht hatten, für die Überweisung in die Idiotenanstalt vorgeschlagen (vgl. Magistrat der Stadt Berlin \& Deputation für die Städtische Irrenpflege 1899). Die langfristige Beobachtung und das Aufschreiben dieser in personalisierten Akten, in der Psychiatrie das entscheidende Instrument zur Wissensproduktion, erschien offensichtlich auch in Schule und Anstalt belastbarer als ein in kurzer Zeit durchgeführter Test. Erst im Rahmen einer gründlichen, zweijährigen Beobachtung im Rahmen des Schulunterrichts sahen sich die Lehrenden und Ärzte in der Lage, eine gesicherte Aussage über den Geisteszustand eines Kindes treffen zu können (vgl. Hintz 1897, insbes. 785). Spätestens mit der Errichtung von Hilfsschulen um 1900 zementierte sich wortwörtlich eine Dreiteilung der „Idiotie“ in „blödsinnig“, „schwachsinnig“ und „schwachbefähigt“, für die dann jeweils eine eigene Institution in Form von Verwahranstalt, Erziehungsanstalt und Hilfsschule existierte. Es waren damit Kategorien und Institutionen in der Welt, die nicht einfach wieder verschwinden würden (vgl. Hofmann 2017). In diesem Kontext erscheinen sowohl Hilfsschule als auch Anstalt eher als Orte der Behandlung und nicht der Diagnose. Die diagnostische Entscheidung über die Überweisung in Anstalt oder Hilfsschule war in den zweijährigen Volksschulbesuch verlagert worden. ${ }^{4}$ Weder in den Hilfsschulen noch in den Anstalten ging allerdings jemand davon aus, dass die Kinder geheilt werden könnten. Das Ziel war vielmehr, „die geistig schwachen dem Leben zuzuführen, sie zu nützlichen Mitgliedern der menschlichen Gesellschaft heranzubilden“" (Piper 1897, 798). Dabei rückte die Vergabe von Diagnosen durch zeitsparende Tests in den Hintergrund. Stattdessen war es nun wichtig, durch Beobachtung des Fortschritts der Kinder Wissen über diejenigen Praktiken hervorzubringen, die das einzelne Kind an das Ziel, arbeitsfähig zu werden, heranführten.

\section{Quellen- und Literaturverzeichnis}

\section{Ungedruckte Quellen}

Landesarchiv Berlin (LAB)

A Rep. 003-04-04 Wittenauer Heilstätten der Stadt Berlin Nr. 2182; 5648; 5668; 5864; 5659; 5861; 5835; 7145 .

\section{Gedruckte Quellen}

Binet, A. \& Simon, T. (1904): Méthodes nouvelles pour le diagnostic du niveau intellectuel des anormaux. In: Lannée Psychologique 11 (1), 191-244. https://doi.org/10.3406/psy.1904.3675.

4 Vielen Dank an meinen Kollegen Stefan Wünsch für diesen Hinweis. 
Binet, A. \& Simon, T. (1905): Mitteilung über die neuen Methoden zur Diagnose des Kretinismus, Schwachsinns und mangelhafter Entwicklung. In: EOS. Zeitschrift für die Erkenntnis und Behandlung Jugendlicher Abnormer, 1. Bd., 247-251.

Bloch, E. (1915): Intelligenzprüfungen an Hilfsschulkindern nach der Methode von Binet-Simon. In: Zeitschrift für Kinderforschung 20 (6), 330-341.

Bobertag, O. (1910): Binets Arbeiten über die intellektuelle Entwicklung des Schulkindes (18941909). In: Zeitschrift für Angewandte Psychologie und Psychologische Sammelforschung. Zugl. Organ d. Instituts für Angewandte Psychologie und Psychologische Sammelforschung, 3. Bd., 230-259.

Bobertag, O. (1911): Über Intelligenzprüfungen (nach der Methode von Binet und Simon). In: Zeitschrift für Angewandte Psychologie und Psychologische Sammelforschung: Zugl. Organ d. Instituts für Angewandte Psychologie und Psychologische Sammelforschung, 4. Bd., 105-211.

Chotzen, F. (1912): Die Intelligenzprüfungsmethode von Binet-Simon bei schwachsinnigen Kindern. In: Zeitschrift für Angewandte Psychologie und Psychologische Sammelforschung. Zugl. Organ d. Instituts für Angewandte Psychologie und Psychologische Sammelforschung, 6. Bd., 412-494.

Ebbinghaus, H. (1897): Über eine neue Methode zur Prüfung geistiger Fähigkeiten und ihre Anwendung bei Schulkindern. In: Zeitschrift für Psychologie und Physiologie der Sinnesorgane, 13. Bd., 401-459.

Falch, E. (1880/81): Ueber die Berechtigung besonderer Klassen bez. Schulen für die leichtesten Formen des Schwachsinns. In: Zeitschrift für das Idiotenwesen 1 (1), 98-106.

Goldscheider, E. (1891): Soltmann: Schrift und Spiegelschrift bei gesunden und kranken Kindern. Festschrift zu Henochs 70. Geburtstag. Berlin, Schumacher, 1890. S. 432-460 (Rezension). In: Zeitschrift für Psychologie und Physiologie der Sinnesorgane, 2. Bd., 414-416.

Goldscheider, E (1892): Zur Physiologie und Pathologie der Handschrift. In: Archiv für Psychiatrie und Nervenkrankheiten 24 (2), 503-525. https://doi.org/10.1007/BF02159941.

Gündel, A. (1896): Zur Klassifizierung der Idioten. Zeitschrift für die Behandlung Schwachsinniger und Epileptischer (Organ der Konferenz für das Idiotenwesen) 16 (5), 73-93.

Hintz, O. (1897). Welche pädagogischen Maßnahmen eignen sich für den Unterricht und die Erziehung solcher Kinder, welche durch die Volksschule nicht genügende Förderung erfahren? In: Pädagogische Zeitung, 26. Bd., 783-786, 821-824.

Ideler, C. \& Magistrat der Stadt Berlin (Hrsg.) (1883): Die städtische Irren-Anstalt zu Dalldorf. I. Geschichte und Verwaltung des städtischen Irrenwesens. II. Beschreibung der neu erbauten IrrenAnstalt zu Dalldorf. Berlin: Springer.

Kannengießer, E. (1895): Beurteilung des kindlichen Geistes durch Spiegelschrift. In: Zeitschrift für die Behandlung Schwachsinniger und Epileptischer (Organ der Konferenz für das Idiotenwesen), 11. Bd., 51-61.

Köster, R. (1904): Die Schrift bei Geisteskranken. Ein Atlas mit 81 Schriftproben. Leipzig: Ambrosius Barth.

Kraepelin, E. (1904a): Psychiatrie. Ein Lehrbuch für Studierende und Ärzte, 1. Bd.: Allgemeine Psychiatrie. 7. Aufl. Leipzig: Ambrosius Barth.

Kraepelin, E. (1904b): Psychiatrie. Ein Lehrbuch für Studierende und Ärzte, 2. Bd.: Klinische Psychiatrie. 7. Aufl. Leipzig: Johann Ambrosius Barth.

Krafft-Ebing, R. von (1888): Lehrbuch der Psychiatrie. Auf klinischer Grundlage für Praktische Ärzte und Studierende. 3. Aufl. Stuttgart: Ferdinand Enke.

Lomer, G. (1913): Ueber graphologische Kennzeichen des Schwachsinns. In: Archiv für Psychiatrie und Nervenkrankheiten 53 (1), 101-173. https://doi.org/10.1007/BF01987361.

Magistrat der Stadt Berlin (1880): Amtlicher Stenographischer Bericht über die Sitzung der Stadtverordnetenversammlung vom 16. März 1880. Stenographische Berichte über die öffentlichen Sitzungen der Stadtverordnetenversammlung der Haupt- und Residenzstadt Berlin, 12. Bd., 156-169. 
Magistrat der Stadt Berlin \& Deputation für die Städtische Irrenpflege (1899): Vorschriften über die Aufnahme von Kindern in die Städtische Idiotenanstalt. Berlin: Emil Schulze.

Piper, H. (1883): Nachrichten über die städtische Irrenanstalt. In: C. Ideler \& Magistrat der Stadt Berlin (Hrsg.): Die städtische Irren-Anstalt zu Dalldorf. Berlin: Springer, 28-30.

Piper, H. (1891): Die Sprachgebrechen bei schwachsinnigen und idiotischen Kindern. In: Monatsschrift für die Gesamte Sprachheilkunde 1 (2), 51-58.

Piper, H. (1893a): Schriftproben von schwachsinnigen resp. idiotischen Kindern. Berlin: Fischers Medicinische Buchhandlung.

Piper, H. (1893b): Zur Aetiologie der Idiotie. Mit einem Vorwort von Dr. W. Sander. Berlin: Fischers Medicinische Buchhandlung.

Piper H. (1895): Statistik über die Sprachgebrechen der in den nachfolgenden Anstalten (Internaten) bezw. Schulen (externaten) befindlichen schwachsinnigen Kinder. In: Zeitschrift für die Behandlung Schwachsinniger und Epileptischer (Organ der Konferenz für das Idiotenwesen) 11 (1), 18-21.

Piper, H. (1897): Idioten und Idiotenanstalten. In: W. Rein (Hrsg.): Enzyklopädisches Handbuch der Pädagogik, 3. Bd.: Griechischer Unterricht - Jünglingsvereine. Langensalza: Hermann Beyer, 788-803.

Sengelmann, H. M. (1885): Idiotophilus. Systematisches Lehrbuch für Idiotenheilpflege, 1. Bd. Norden: Soltau.

Treitel, L. (1893): Ueber das Schreiben mit der linken Hand und Schreibstörungen, besonders auf Grund von Schuluntersuchungen. In: Deutsche Zeitschrift für Nervenheilkunde 4 (3/4), 277-290. https://doi.org/10.1007/BF01665291.

Ziehen, T. (1894): Psychiatrie für Ärzte und Studirende. Berlin: Friedrich Wreden.

\section{Literatur}

Ankele, M. (2012): Ausdrucksbewegungen im Fokus des psychiatrischen Blicks um 1900. Aspekte einer „Diskursivierung des Alltäglichen“. In: M. Wernli (Hrsg.): Wissen und Nicht-Wissen in der Klinik. Dynamiken der Psychiatrie um 1900. Bielefeld: transcript, 87-114.

Borck, C. \& Schäfer, A. (2015): Das psychiatrische Aufschreibesystem. In: C. Borck \& A. Schäfer (Hrsg.): Das psychiatrische Aufschreibesystem. Paderborn: Wilhelm Fink, 7-28.

Bühler, P. (2017): „Diagnostik“ und „praktische Behandlung“. Die Entstehung der therapeutischen Funktion der Schule. In: R. Reichenbach \& P. Bühler (Hrsg.): Fragmente zu einer pädagogischen Theorie der Schule. Erziehungswissenschaftliche Perspektiven auf eine Leerstelle. Weinheim: Beltz Juventa, 176-195.

Canguilhem, G. (1989): Grenzen medizinischer Rationalität. Historisch-epistemologische Untersuchungen. Tübingen: edition diskord.

Carson, J. (2014): Mental testing in the early twentieth century: internationalizing the mental testing story. In: History of Psychology, 17. Jg., 249-255. https://doi.org/10.1037/a0037475.

Engstrom, E. J. (2003): Clinical Psychiatry in Imperial Germany. A History of Psychiatric Practice. London: Ithaca.

Engstrom, E. J. (2005): Die Ökonomie klinischer Inskription. Zu diagnostischen und nosologischen Schreibpraktiken in der Psychiatrie. In: C. Borck \& A. Schäfer (Hrsg.): Psychographien. Zürich: Diaphanes, 219-240.

Gstach, J. (2015): Kretinismus und Blödsinn. Zur fachlich-wissenschaftlichen Entdeckung und Konstruktion von Phänomenen der geistig-mentalen Auffälligkeit zwischen 1780 und 1900 und deren Bedeutung für Fragen der Erziehung und Behandlung. Bad Heilbrunn: Klinkhardt.

Hagner, M. (2000): Homo cerebralis. Der Wandel vom Seelenorgan zum Gehirn. Frankfurt/M.: Insel Verlag.

Hess, V. (2010): Formalisierte Beobachtung. Die Genese der modernen Krankenakte am Beispiel der Berliner und Pariser Medizin (1725-1830). In: Medizinhistorisches Journal 45 (3/4), 293-340. 


\section{2| Jona Garz}

Hofmann, M. (2017): Schwachbegabt, schwachsinnig, blödsinnig. Kategorisierung geistig beeinträchtiger Kinder um 1900. In: Bildungsgeschichte. International Journal on the Historiography of Education 7 (2), 142-156.

Kammer, S. (2013): Autographien als Krankheit. Physiologie und Pathologie der Handschrift um 1900. In: Y. Wübben \& C. Zelle (Hrsg.): Krankheit schreiben. Aufzeichnungsverfahren in Medizin und Literatur. Göttingen: Wallstein, 225-247.

Ledebur, S. (2011): Schreiben und Beschreiben. Zur epistemischen Funktion von psychiatrischen Krankenakten, ihrer Archivierung und deren Übersetzung in Fallgeschichten. In: Berichte zur Wissenschaftsgeschichte 34 (2), 102-124. https://doi.org/10.1002/bewi.201101473.

Ledebur, S. (2013): Sehend schreiben, schreibend sehen. Vom Aufzeichnen psychischer Phänomene in der Psychiatrie. In: Y. Wübben \& C. Zelle (Hrsg.): Krankheit schreiben. Aufzeichnungsverfahren in Medizin und Literatur. Göttingen: Wallstein, 82-108.

Ledebur, S. (2015): Verstetigen eines Moments. Zum Verfahren des stenographischen Protokollierens in der Psychiatrie. In: C. Borck \& A. Schäfer (Hrsg.): Das psychiatrische Aufschreibesystem. Paderborn: Wilhelm Fink, 29-54.

Linn, R. L. (2001): A Century of Standardized Testing. Controversies and Pendulum Swings. In: Educational Assessment 7 (1), 29-38.

Opitz, F. (1952): Sie wirkten in Berlin. Erinnerungsschrift anläßlich des Kongresses der Lehrer und Erzieher in Berlin Pfingsten 1952 überreicht. Berlin: Berliner Verb. der Lehrer und Erzieher.

Rheinberger, H.-J. (2007): Kulturen des Experiments. In: Berichte Zur Wissenschaftsgeschichte 30 (2), 135-144. https://doi.org/10.1002/bewi.200701248.

Schäfer, A. (2005): Lebendes Dispositiv. Hand beim Schreiben. In: C. Borck \& A. Schäfer (Hrsg.): Psychographien. Zürich: Diaphanes, 41-265.

Wittmann, B. (2005): Zeichnen im Dunkeln. Psychophysiologie einer Kulturtechnik um 1900. In: W. Busch, O. Jehle \& C. Meister (Hrsg.): Randgänge der Zeichnung, München: Wilhelm Fink, 2007, 165-186. 